\title{
Asymptotic Stability of Uncertain Lagrangian Systems with Prescribed Transient Response
}

\author{
Christos K. Verginis and Dimos V. Dimarogonas
}

\begin{abstract}
This paper considers the asymptotic tracking problem for 2nd-order nonlinear Lagrangian systems subject to predefined constraints for the system response, such as maximum overshoot or minimum convergence rate. In particular, by employing discontinuous adaptive control protocols and nonsmooth analysis, we extend previous results on funnel control to guarantee at the same time asymptotic trajectory tracking from all the initial conditions that are compliant with the given funnel. The considered system contains parametric and structural uncertainties, with no boundedness or approximation/parametric factorization assumptions. The response of the closed loop system is solely determined by the predefined funnel and is independent from the control gain selection. Finally, simulation results verify the theoretical findings.
\end{abstract}

\section{INTRODUCTION}

Analysis and control of uncertain systems consists one of the dominant topics in control theory and design, since it has numerous applications in real-world scenarios, where the systems are affected by modeling uncertainties and external disturbances. One of the main control methodologies developed to tackle uncertain systems is adaptive control, where a time-varying gain is employed to estimate/compensate the system uncertainties [1]-[3], [3]-[5].

A well-studied instance of adaptive control is funnel control, where the goal is the confinement of the system state to a predefined funnel [6]-[8]. Funnel control is a high-gain control scheme that usually does not use any information on the system model, and has had numerous applications during the last years. Examples include chemical reactors [9], robotic manipulation [10], [11], vehicle platooning [12], temporal logic planning [13], and multi-agent systems [14]-[16]. Intuitively, funnel control design is based on an adaptive gain, which increases to infinity as the system state approaches the funnel boundary, "pushing" in that way the state to remain in the funnel, by also compensating for the (possibly unknown) dynamics. The intuition behind funnel control is the incorporation of an adaptive gain in the control scheme, which increases (in absolute value) as the system's output reaches the funnel's boundary. In that way, the system's output is "pushed" to always remain inside the funnel. Funnel control has been developed for both linear (e.g., [17]) and nonlinear systems (e.g., [6], [8], [18]),

The authors are with the School of Electrical Engineering and Computer Science, KTH Royal Institute of Technology, SE-100 44, Stockholm, Sweden. Email: $\{$ cverginis, dimos\}akth.se. This work was supported by the H2020 ERC Starting Grant BUCOPHSYS, the European Union's Horizon 2020 Research and Innovation Programme under the GA No. 731869 (Co4Robots), the Swedish Research Council (VR), the Knut och Alice Wallenberg Foundation (KAW) and the Swedish Foundation for Strategic Research (SSF). involving parametric (e.g., [10]) as well as structural (e.g., [8]) dynamic uncertainties, for a wide class of systems. It was also combined with a bang-bang controller for SISO systems in [19] and input saturation in [20].

Traditionally, funnel control guarantees that the state is confined in a predefined funnel, without necessarily converging to zero, i.e., asymptotic stability is not taken into account. As a workaround, if the funnel is specified by the control designer, it can "shrink" asymptotically, i.e., become arbitrarily narrow around zero as time grows to infinity, achieving thus "practical stability". This, however, might yield undesired large inputs due to the small funnel values, and can be problematic in real-time systems. On the other hand, by achieving asymptotic stability for the state, the funnel is not needed to converge close to zero, and can be used in order to encode just transient constraints for the system. Asymptotic tracking subject to funnel transient constraints has been considered in several works [10], [15], [17], [21]; [15], [17], [21] consider linear systems, whereas [10] assumes a known model structure, with the uncertainties being only parametric; The aforementioned works cannot be extended in a straightforward manner to cases where the dynamic terms are nonlinear and have both parametric and structural uncertainties. In addition, a class of systems for which funnel control has not been considered in the related works is the non-smooth type, i.e., systems with discontinuous right-hand side terms (e.g., friction terms). Such models are motivated by real-time systems, where several dynamic terms (e.g., friction) are accurately modeled by discontinuous functions of the state.

In this paper, we consider the asymptotic tracking control problem subject to transient constraints imposed by a predefined funnel for 2nd-order Lagrangian systems with uncertain and nonsmooth nonlinear terms. More specifically, we design a discontinuous adaptive control protocol that guarantees confinement of the state in the given funnel as well as asymptotic stability, from all initial conditions that satisfy the initial funnel constraints, independently of the system model. The proposed control law is of low complexity and does not incorporate any information on the system model, and no boundedness assumptions or growth conditions are considered. Discontinuous control schemes have been considered in the related literature, mostly for stabilization of nonholonomic systems, e.g., [22]-[26], without taking into account transient funnel constraints. Moreover, many of these works deal with parametric model uncertainties or assume uniform boundedness and/or growth conditions on the unknown dynamical terms. Discontinuous control schemes for 
system uncertainties are also used in our works [27], [28] that tackle multi-agent problems. The model uncertainties in these works, however, are restricted to uniformly bounded disturbances and terms bounded by the state norm.

The rest of the paper is structured as follows. Section II introduces preliminary background notation. Section III describes the tackled problem and Section IV provides the proposed control protocol and the stability analysis. Finally, simulation results are given in Section $\mathrm{V}$ and Section VI concludes the paper.

\section{NOTATION AND PRELIMINARIES}

\section{A. Notation}

The sets of real and positive real numbers are denoted by $\mathbb{R}$ and $\mathbb{R}_{>0}$, respectively; $\|x\|_{1}$ and $\|x\|$ denote the 1 - and 2-norm, respectively, of a vector $x \in \mathbb{R}^{n}$; The open and closed balls with radius $\delta$, centered at $x \in \mathbb{R}^{n}$, are denoted by $\mathcal{B}(x, \delta)$ and $\overline{\mathcal{B}}(x, \delta)$, respectively. The sign function is defined as $\operatorname{sgn}(x)=\{-1,0,1\}$ for $x<0, x=$ $0, x>0$, respectively; its vector counterpart is defined as $\operatorname{sgn}(x)=\left[\operatorname{sgn}\left(x_{1}\right), \ldots, \operatorname{sgn}\left(x_{n}\right)\right]^{\top} \in \mathbb{R}^{n}$, for $x=$ $\left[x_{1}, \ldots, x_{n}\right]^{\top} \in \mathbb{R}^{n}$. Given a function $f: \mathbb{R}^{n} \rightarrow \mathbb{R}^{k}$, its Filippov regularization is defined as [29] $\mathrm{K}[f](x):=$ $\cap_{\delta>0} \cap_{\mu(\bar{N})=0} \overline{\mathrm{co}}(f(\mathcal{B}(x, \delta) \backslash \bar{N}), t)$, where $\bigcap_{\mu(\bar{N})=0}$ is the intersection over all sets $\bar{N}$ of Lebesgue measure zero, and $\overline{\mathrm{co}}(E)$ is the convex closure of the set $E$. The Filippov regularization of $\operatorname{sgn}(x) \in \mathbb{R}$ is denoted by $\mathrm{K}[\operatorname{sgn}](x)=$ $\operatorname{SGN}(x)$ where $\operatorname{SGN}(x)=-1$, if $x<0, \operatorname{SGN}(x)=1$, if $x>0$, and $\operatorname{SGN}(x) \in[-1,1]$, if $x=0$. For a vector $x=\left[x_{1}, \ldots, x_{n}\right]^{\top} \in \mathbb{R}^{n}$, we use the notation $\operatorname{SGN}(x):=$ $\left[\operatorname{SGN}\left(x_{1}\right), \ldots, \operatorname{SGN}\left(x_{n}\right)\right]^{\top} \in \mathbb{R}^{n}$.

\section{B. Nonsmooth Analysis}

Consider the following differential equation with a discontinuous right-hand side:

$$
\dot{x}=f(x, t),
$$

where $f: \mathcal{D} \times\left[t_{0}, \infty\right) \rightarrow \mathbb{R}^{n}, \mathcal{D} \subset \mathbb{R}^{n}$, is Lebesgue measurable and locally essentially bounded.

Definition 1 (Def. 1 of [30]): A function $x:\left[t_{0}, t_{1}\right) \rightarrow$ $\mathbb{R}^{n}$, with $t_{1}>t_{0}$, is called a Filippov solution of (1) on $\left[t_{0}, t_{1}\right)$ if $x(t)$ is absolutely continuous and if, for almost all $t \in\left[t_{0}, t_{1}\right)$, it satisfies $\dot{x}(t) \in \mathrm{K}[f](x, t)$, where $\mathrm{K}[f](x, t)$ is the Filippov regularization of $f(x, t)$.

Lemma 1 (Lemma 1 of [30]): Let $x(t)$ be a Filippov solution of (1) and $V: \mathcal{D} \times\left[t_{0}, t_{1}\right) \rightarrow \mathbb{R}$ be a locally Lipschitz, regular function ${ }^{1}$. Then $V(x(t), t)$ is absolutely continuous, $\dot{V}(x(t), t)=\frac{\partial}{\partial t} V(x(t), t)$ exists almost everywhere (a.e.), i.e., for almost all $t \in\left[t_{0}, t_{1}\right)$, and $\dot{V}(x(t), t) \stackrel{\text { a.e }}{\in} \dot{\widetilde{V}}(x(t), t)$, where $\dot{\tilde{V}}:=\cap_{\xi \in \partial V(x, t)} \xi^{\top}\left[\mathrm{K}[f](x, t)^{\top}, 1\right]^{\top}$, and $\partial V(x, t)$ is Clarke's generalized gradient at $(x, t)$ [30].

Theorem 1 (Corollary 2 of [30]): For (1), let $\mathcal{D} \subset \mathbb{R}^{n}$ be an open and connected set containing $x=0$ and suppose that $f$ is Lebesgue measurable and $x \mapsto f(x, t)$ is essentially

\footnotetext{
${ }^{1}$ See [30] for a definition of regular functions.
}

locally bounded, uniformly in $t$. Let $V: \mathcal{D} \times\left[t_{0}, t_{1}\right) \rightarrow \mathbb{R}$ be locally Lipschitz and regular such that $W_{1}(x) \leq V(x, t) \leq$ $W_{2}(x), \forall t \in\left[t_{0}, t_{1}\right), x \in \mathcal{D}$, and $z \leq-W(x(t)), \forall z \in$ $\dot{\tilde{V}}(x(t), t), t \in\left[t_{0}, t_{1}\right), x \in \mathcal{D}$, where $W_{1}$ and $W_{2}$ are continuous positive definite functions and $W$ is a continuous positive semi-definite function on $\mathcal{D}$. Choose $r>0$ and $c>0$ such that $\overline{\mathcal{B}}(0, r) \subset \mathcal{D}$ and $c<\min _{\|x\|=r} W_{1}(x)$. Then for all Filippov solutions $x:\left[t_{0}, t_{1}\right) \rightarrow \mathbb{R}^{n}$ of (1), with $x\left(t_{0}\right) \in \mathbb{D}:=\left\{x \in \overline{\mathcal{B}}(0, r): W_{2}(x) \leq c\right\}$, it holds that $t_{1}=\infty, x(t) \in \mathbb{D}, \forall t \in\left[t_{0}, \infty\right)$, and $\lim _{t \rightarrow \infty} W(x(t))=0$.

\section{PROBLEM FORMULATION}

Consider a MIMO Lagrangian system of the form

$$
M(x) \ddot{x}+f(x, \dot{x})+g(x)=u,
$$

where $x:=\left[x_{1}, \ldots, x_{n}\right]^{\top} \in \mathbb{R}^{n}, \dot{x}:=\left[\dot{x}_{1}, \ldots, \dot{x}_{n}\right]^{\top} \in \mathbb{R}^{n}$ is the system's state, which is available for measurement, $M: \mathbb{R}^{n} \rightarrow \mathbb{R}^{n \times n}$ is a positive definite inertia term, $f$ : $\mathbb{R}^{2 n} \rightarrow \mathbb{R}^{n}$ is a term representing Coriolis and frictionterms, $g: \mathbb{R}^{n} \rightarrow \mathbb{R}^{n}$ is a gravity term, and $u \in \mathbb{R}^{n}$ is the control input to be designed. The terms $M(\cdot), g(\cdot)$ are assumed to be continuous everywhere, while the term $f(\cdot)$ is assumed to be Lebesgue measurable and locally essentially bounded, which resembles friction-like terms that might be discontinuous functions of the state. This is rigorously stated in the following assumption:

Assumption 1: The maps $x \mapsto M(x): \mathbb{R}^{n} \rightarrow \mathbb{R}^{n \times n}, x \mapsto$ $g(x): \mathbb{R}^{n} \rightarrow \mathbb{R}^{n}$ are continuous and the map $f: \mathbb{R}^{2 n} \rightarrow \mathbb{R}^{n}$ is Lebesgue measurable and locally bounded.

Moreover, since (2) represents a Lagrangian system, the inertia term satisfies the following property:

Property 1: $M(x)$ is symmetric and positive definite, $\forall x \in \mathbb{R}^{n}$, and $0<\underline{m} I_{n} \leq M(x) \leq \bar{m} I_{n}, \forall x \in \mathbb{R}^{n}$, where $\underline{m}, \bar{m}$ are unknown positive and finite constants.

The aforementioned property poses a controllability condition (similar to the ones considered in a variety of works, e.g., [2], [3], [6], [18]).

The control objective is the asymptotic tracking of a desired bounded trajectory $x_{\mathrm{d}}:=\left[x_{1, \mathrm{~d}}, \ldots, x_{n, \mathrm{~d}}\right]:\left[t_{0}, \infty\right) \rightarrow$ $\mathbb{R}^{n}$, with bounded first and second derivatives, i.e., $\left\|x_{\mathrm{d}}(t)\right\|<$ $\bar{x}_{\mathrm{d}, 0} \leq \bar{x}_{\mathrm{d}},\left\|\dot{x}_{\mathrm{d}}(t)\right\|<\bar{x}_{\mathrm{d}, 1} \leq \bar{x}_{\mathrm{d}},\left\|\ddot{x}_{\mathrm{d}}(t)\right\|<\bar{x}_{\mathrm{d}, 2} \leq$ $\bar{x}_{\mathrm{d}}, t \in\left[t_{0}, \infty\right)$, for unknown finite constants $\bar{x}_{\mathrm{d}, j}>0$, $j \in\{0,1,2\}$, and $\bar{x}_{\mathrm{d}}:=\max _{j \in\{0,1,2\}}\left\{\bar{x}_{\mathrm{d}, j}\right\}$. Moreover, as discussed in Section I, we aim at imposing a certain predefined behavior for the transient response of the system. More specifically, motivated by funnel control techniques [6], [8], [18], given $n$ predefined funnels, described by the smooth functions (also called performance functions in [6]) $\rho_{p_{i}}:\left[t_{0}, \infty\right) \rightarrow\left[\underline{\rho}_{p_{i}}, \bar{\rho}_{p_{i}}\right] \subset \mathbb{R}_{>0}$, where $\underline{\rho}_{p_{i}}, \bar{\rho}_{p_{i}}$ are the positive lower and upper bounds, respectively, we aim at guaranteeing that ${ }^{2}-\rho_{p_{i}}(t)>x_{i}(t)-x_{i, \mathrm{~d}}(t)>\rho_{p_{i}}(t), \forall t \in$ $\left[t_{0}, \infty\right)$, given that $-\rho_{p_{i}}\left(t_{0}\right)>x_{i}\left(t_{0}\right)-x_{i, \mathrm{~d}}\left(t_{0}\right)>\rho_{p_{i}}\left(t_{0}\right)$, $\forall i \in\{1, \ldots, n\}$. These functions can encode maximum overshoot or convergence rate properties. Note that, compared to the majority of the related works on funnel control

\footnotetext{
${ }^{2}$ The results can be extended to non-symmetric funnels.
} 
(e.g., [6], [8], [18]), we do not require arbitrarily small final values $\lim _{t \rightarrow \infty} \rho_{p_{i}}(t)$, which would achieve convergence of $x_{i}(t)-x_{i, \mathrm{~d}}(t)$ arbitrarily close to zero, since one of the objectives is actual asymptotic stability. Formally, the problem statement is the following:

Problem 1: Consider the system (2) and let a desired trajectory $x_{\mathrm{d}}:\left[t_{0}, \infty\right) \rightarrow \mathbb{R}^{n}$ as well as $n$ prescribed funnels, described by $\rho_{p_{i}}:\left[t_{0}, \infty\right) \rightarrow\left[\rho_{p_{i}}, \bar{\rho}_{p_{i}}\right]$. Design a control protocol $u \in \mathbb{R}^{n}$ such that $\lim _{t \rightarrow \infty}\left(x_{i}(t)-x_{i, \mathrm{~d}}(t)\right)=0$ and $-\rho_{p_{i}}(t)>x_{i}(t)-x_{i, \mathrm{~d}}(t)>\rho_{p_{i}}(t), \forall i \in\{1, \ldots, n\}$, $t \in\left[t_{0}, \infty\right)$, and all closed loop signals remain bounded.

\section{MAIN RESULTS}

Our solution to Problem 1 is based on the error transformation proposed in [6], which converts the constrained error behavior $-\rho_{p_{i}}(t)>x_{i}(t)-x_{i, \mathrm{~d}}(t)>\rho_{p_{i}}(t)$ to an unconstrained one. More specifically, we define the errors

$$
e_{p}:=\left[e_{p_{1}}, \ldots, e_{p_{n}}\right]^{\top}:=x-x_{\mathrm{d}},
$$

as well as the error transformations $\varepsilon_{p_{i}} \in \mathbb{R}$ according to:

$$
e_{p_{i}}=\rho_{p_{i}} T\left(\varepsilon_{p_{i}}\right), \forall i \in\{1, \ldots, n\},
$$

where $T: \mathbb{R} \rightarrow(-1,1)$ is a smooth, strictly increasing function, with $T(0)=0$. Since $T$ is increasing, the inverse mapping $T^{-1}:(-1,1) \rightarrow \mathbb{R}$ is well-defined, and

$$
\lim _{z \rightarrow-\infty} T(z)=-1 \quad \lim _{z \rightarrow+\infty} T(z)=1
$$

and hence, if $\varepsilon_{p_{i}}$ remains bounded in a compact set, the desired funnel objective $-\rho_{p_{i}}(t)<e_{p_{i}}(t)<\rho_{p_{i}}(t)$ is achieved, $\forall i \in\{1, \ldots, n\}$. We further require that

$$
|z| \leq\left|\frac{\partial T^{-1}(z)}{\partial z} T^{-1}(z)\right|, \quad \forall z \in(-1,1) .
$$

A possible choice that satisfies the aforementioned specifications is $T(z):=\frac{\exp (z)-1}{\exp (z)+1}$.

From (4), we obtain $\varepsilon_{p_{i}}=T^{-1}\left(\frac{e_{p_{i}}}{\rho_{p_{i}}}\right)$, which, after differentiation, becomes $\dot{\varepsilon}_{p_{i}}=r_{p_{i}} \rho_{p_{i}}^{-1}\left(\dot{x}_{i}-\dot{x}_{i, \mathrm{~d}}-\dot{\rho}_{p_{i}} e_{p_{i}} \rho_{p_{i}}^{-1}\right)$, or, in stack vector form,

$$
\dot{\varepsilon}_{p}=r_{p} \rho_{p}^{-1}\left(\dot{x}-\dot{x}_{\mathrm{d}}-\dot{\rho}_{p} \rho_{p}^{-1} e_{p}\right),
$$

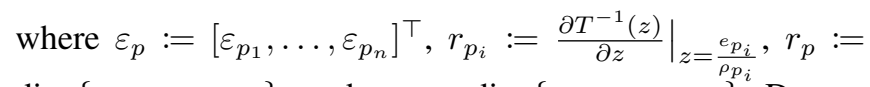
$\operatorname{diag}\left\{r_{p_{1}}, \ldots, r_{p_{n}}\right\}$, and $\rho_{p}:=\operatorname{diag}\left\{\rho_{p_{1}}, \ldots, \rho_{p_{n}}\right\}$. Due to the increasing property of $T(\cdot)$ (and hence of $T^{-1}(\cdot)$ ), it holds that $r_{p}$ is positive definite, and thus in order to render $\dot{\varepsilon}_{p}$ negative a choice for a desired value for $\dot{x}$ is

$$
v_{\mathrm{d}}:=\dot{x}_{\mathrm{d}}+\dot{\rho}_{p} \rho_{p}^{-1} e_{p}-k_{p} r_{p} \varepsilon_{p},
$$

where $k_{p}>0$ is a positive scalar constant gain. Since, however, $\dot{x}$ is not the system's input, we follow a backsteppinglike methodology and define the error

$$
e_{v}:=\left[e_{v_{1}}, \ldots, e_{v_{n}}\right]^{\top}:=\dot{x}-v_{\mathrm{d}} .
$$

Next, we proceed in a similar manner and define a funnel for each $e_{v_{i}}, i \in\{1, \ldots, n\}$, described by the functions $\rho_{v_{i}}:\left[t_{0}, \infty\right) \rightarrow\left[\underline{\rho}_{v_{i}}, \bar{\rho}_{v_{i}}\right] \subset \mathbb{R}_{>0}$, where $\underline{\rho}_{v_{i}}, \bar{\rho}_{v_{i}}$ are the positive lower and upper bounds, respectively, with the constraint $\rho_{v_{i}}\left(t_{0}\right)>\left|e_{v_{i}}\left(t_{0}\right)\right|, i \in\{1, \ldots, n\}$. Note that $e_{v_{i}}\left(t_{0}\right)=\dot{x}\left(t_{0}\right)-v_{\mathrm{d}}\left(t_{0}\right)$ can be calculated at $t=t_{0}$ since it is a function of the state, the funnel functions and the desired trajectory profile. Moreover, note that the functions $\rho_{v_{i}}$ represent an artificial funnel, in the sense that they are not part of the given specification (as $\rho_{p_{i}}$ ). The design constraints they need to satisfy concern boundedness, positivity, and initial compliance with respect to the respective errors, i.e., $\rho_{v_{i}}\left(t_{0}\right)>\left|e_{v_{i}}\left(t_{0}\right)\right|, \forall i \in\{1, \ldots, n\}$.

Next, we define the open set $\mathcal{D}_{u, t}:=\{(x, \dot{x}, t) \in$ $\mathbb{R}^{2 n} \times\left[t_{0}, \infty\right): \rho_{p}(t)^{-1} e_{p}(t) \in(-1,1)^{n}, \rho_{v}(t)^{-1} e_{v}(t) \in$ $\left.(-1,1)^{n}\right\}$, and design the control law $u: \mathcal{D}_{u, t} \rightarrow \mathbb{R}^{n}$ as

$$
\begin{aligned}
u= & -k_{v_{2}} \rho_{v}^{-1} \operatorname{sgn}\left(r_{v} \varepsilon_{v}\right)\left(k_{v_{3}}\left\|r_{p} \varepsilon_{p}\right\|_{1}+k_{v_{4}} \hat{d}\right) \\
& -k_{v_{1}} \rho_{v}^{-1} r_{v} \varepsilon_{v}
\end{aligned}
$$

where $\rho_{v}:=\operatorname{diag}\left\{\rho_{v_{1}}, \ldots, \rho_{v_{n}}\right\}, \varepsilon_{v}:=\left[\varepsilon_{v_{1}}, \ldots, \varepsilon_{v_{n}}\right]^{\top}$, $\varepsilon_{v_{i}}:=T^{-1}\left(\frac{e_{v_{i}}}{\rho_{v_{i}}}\right), r_{v}:=\operatorname{diag}\left\{r_{v_{1}}, \ldots, r_{v_{n}}\right\}, r_{v_{i}}:=$ $\left.\frac{\partial T^{-1}(z)}{\partial z}\right|_{z=\frac{e_{v_{i}}}{\rho_{v_{i}}}}, k_{v_{i}}>0, i \in\{1, \ldots, 4\}$ are positive scalar constant gains, and $\hat{d}$ is an adaptive variable gain, subject to the constraint $\hat{d}\left(t_{0}\right) \geq 0$, and dynamics

$$
\dot{\hat{d}}=\gamma_{d}\left\|r_{v} \varepsilon_{v}\right\|_{1}
$$

where $\gamma_{d}>0$ is a positive constant gain.

Remark 1: The control design procedure follows closely the prescribed performance backstepping-like methodology of the previous works [11]-[13], [16], introduced in [31]. The desired signals and control laws in these works consist only of proportional terms with respect to the transformed errors $\varepsilon_{p}, \varepsilon_{v}$, which are guaranteed to be ultimately bounded. In this work, the incorporation of the extra terms in (8) and (10) achieves convergence of the transformed errors to zero, guaranteeing thus asymptotic stability.

Remark 2: Note that no information regarding the dynamic model is incorporated in the control protocol (3)(11). All the necessary signals consist of the funnel terms $\rho_{p}, \rho_{v}$ and of known functions of the state and the desired trajectory $x_{\mathrm{d}}$. Furthermore, no a-priori gain tuning is needed and, as the next theorem states, the solution of Problem 1 is guaranteed from all initial conditions that satisfy $-\rho_{p_{i}}\left(t_{0}\right)>$ $x_{i}\left(t_{0}\right)-x_{i, \mathrm{~d}}\left(t_{0}\right)>\rho_{p_{i}}\left(t_{0}\right), \forall i \in\{1, \ldots, n\}$. As will be revealed subsequently, the adaptive gain $\hat{d}$ compensates the unknown dynamic terms, which are proven to be bounded due to the confinement of the state in the prescribed funnels.

The correctness of the control protocol (3)-(11) is shown in the next theorem.

Theorem 2: Consider a system subject to the dynamics (2), Assumption 1, Property 1, as well as a desired trajectory $x_{\mathrm{d}}$ and funnels $\rho_{p_{i}}$ as described in Problem 1. Then the control protocol (3)-(11) guarantees the solution of Problem 1 from all initial conditions that satisfy $-\rho_{p_{i}}\left(t_{0}\right)>x_{i}\left(t_{0}\right)-$ $x_{i, \mathrm{~d}}\left(t_{0}\right)>\rho_{p_{i}}\left(t_{0}\right), \forall i \in\{1, \ldots, n\}$.

Proof: The intuition of the subsequent proof is as follows: We first show the existence of at least one Filippov 
solution of the closed-loop system in $\mathcal{D}_{u, t}$ for a time interval $I \subseteq\left[t_{0}, \infty\right)$. Next, we prove that for any of these solutions, the state remains bounded in $I$ by bounds independent of the endpoint of $I$. Hence, the dynamic terms of (2) are also upper bounded by a term, which we aim to compensate via the adaptation gain $\hat{d}$.

We start by defining some terms that will be used in the subsequent analysis: $M_{p}:=$ $\max _{i \in\{1, \ldots, n\}}\left\{\bar{\rho}_{p_{i}}\right\}, \quad m_{p}:=\min _{i \in\{1, \ldots, n\}}\left\{\underline{\rho}_{p_{i}}\right\}$, $M_{\dot{p}}:=\max _{i \in\{1, \ldots, n\}}\left\{\sup _{t \geq t_{0}}\left\{\left|\dot{\rho}_{p_{i}}(t)\right|\right\}\right\}, \quad M_{v}:=$ $\max _{i \in\{1, \ldots, n\}}\left\{\bar{\rho}_{v_{i}}\right\}, m_{v}:=\min _{i \in\{1, \ldots, n\}}\left\{\underline{\rho}_{v_{i}}\right\}, \underline{\lambda}:=\frac{1}{M_{v}^{2} \bar{m}}$, $\beta:=\left(k_{v_{2}} k_{v_{4}} \underline{\lambda}\right)^{-1}, \underline{r}_{p}:=\inf _{z \in(-1,1)} \frac{\partial T^{-1}(z)}{\partial z}$, where $\bar{m}$ was introduced in Property 1 . Note that all the aforementioned terms are strictly positive. In particular, $\underline{\lambda}$ is a lower bound for $\left\|\rho_{v}^{-1} M(x)^{-1} \rho_{v}^{-1}\right\|$ and is strictly positive due to the definition of the funnels $\rho_{v}$ and Property 1 , and $\underline{r}_{p}$ is positive due to the strictly increasing property of $T(\cdot)$ and hence of $T^{-1}(\cdot)$. Moreover, in view of (5), it holds that $\arg \inf _{z \in(-1,1)} \frac{\partial T^{-1}(z)}{\partial z} \in(-1,1)$. By employing (10), (11), the closed loop system becomes

$$
\begin{aligned}
& \ddot{x} \in M(x)^{-1}(\mathrm{~K}[u](x, \dot{x}, t)-\mathrm{K}[f](x, \dot{x})-g(x)), \\
& \dot{\hat{d}}=\gamma_{d}\left\|r_{v} \varepsilon_{v}\right\|_{1},
\end{aligned}
$$

where $\mathrm{K}[F](x, \dot{x}), \mathrm{K}[u](x, \dot{x}, t)$ are the Filippov regularizations of the respective terms. For $u$ specifically, $\mathrm{K}[u](x, \dot{x}, t)$ is formed by substituting the term $\operatorname{sgn}\left(r_{v} \varepsilon_{v}\right)$ with $\operatorname{SGN}\left(r_{v} \varepsilon_{v}\right)$. Define now $\widetilde{x}:=\left[x^{\top}, \dot{x}^{\top}, \hat{d}\right] \in \mathbb{R}^{2 n+1}$ and consider the open set $\mathcal{D}_{c}:=\left\{(\widetilde{x}, t) \in \mathbb{R}^{2 n+1} \times\left[t_{0}, \infty\right)\right.$ : $\left.(x, t) \in \mathcal{D}_{u, t}\right\}$. Since $\rho_{p_{i}}\left(t_{0}\right)>\left|e_{p_{i}}\left(t_{0}\right)\right|$ and $\rho_{v_{i}}\left(t_{0}\right)>$ $\left|e_{v_{i}}\left(t_{0}\right)\right|, \forall i \in\{1 \ldots, n\}$, the set $\mathcal{D}_{c}$ is nonempty. Moreover, the right hand-side of (12) is Lebesgue measurable and locally bounded in $\widetilde{x}$ over the set $\left\{\widetilde{x}:(\widetilde{x}, t) \in \mathcal{D}_{c}\right\}$, and Lebesgue measurable in $t$ over the set $\left\{t:(\widetilde{x}, t) \in \mathcal{D}_{c}\right\}$. Hence, according to Prop. 3 of [32], for each initial condition $\left(\widetilde{x}\left(t_{0}\right), t_{0}\right) \in \mathcal{D}_{c}$, there exists at least one Filippov solution $\widetilde{x}(t)$ of (12), defined in $I:=\left[t_{0}, t_{\max }\right)$, where $t_{\max }>$ $t_{0}$, such that $(\widetilde{x}(t), t) \in \mathcal{D}_{c}, \forall t \in I$. By applying the transformation $T(\cdot)^{-1}$, we conclude the existence of the respective Filippov solutions $\varepsilon_{p}(t), \varepsilon_{v}(t) \in \mathbb{R}^{n}, \forall t \in I$. Let now $\widetilde{x}\left(t_{0}\right)$ denote the initial condition of the system (12) satisfying $\left(\widetilde{x}\left(t_{0}\right), t_{0}\right) \in \mathcal{D}_{c}$ and consider the family of Filippov solutions starting from $\widetilde{x}\left(t_{0}\right)$ denoted by the set $\mathfrak{X}$. Note that, although not explicitly stated, $t_{\max }$ and $I$ might be different for each solution in $\mathfrak{X}$. We aim to prove that all $\varepsilon_{p}(t)$ and $\varepsilon_{v}(t)$ are bounded and that converge to zero, for all $\widetilde{x}(t) \in \mathfrak{X}$. In view of $\mathcal{D}_{c}$, for all $\widetilde{x}(t) \in \mathfrak{X}$ it holds that

$$
\left|e_{p_{i}}(t)\right|<\bar{\rho}_{p_{i}}, \quad\left|e_{v_{i}}(t)\right|<\bar{\rho}_{v_{i}},
$$

$\forall t \in I$, where $\bar{\rho}_{p_{i}}$ and $\bar{\rho}_{v_{i}}$ are the upper bounds of $\rho_{p_{i}}(t)$ and $\rho_{v_{i}}(t)$, respectively, $\forall i \in\{1, \ldots, n\}$. Consider now the Lyapunov function $V_{p}:=\frac{1}{2}\left\|\varepsilon_{p}\right\|^{2}$, for which it holds, in view of (7) - (9), and (13)

$$
\begin{aligned}
\dot{V}_{p} & =\varepsilon_{p}^{\top} r_{p} \rho_{p}^{-1}\left(\dot{x}-\dot{x}_{\mathrm{d}}-\dot{\rho}_{p} \rho_{p}^{-1} e_{p}\right) \\
& \leq-\frac{k_{p}}{M_{p}}\left\|r_{p} \varepsilon_{p}\right\|^{2}+\frac{M_{v}}{m_{p}}\left\|r_{p} \varepsilon_{p}\right\|
\end{aligned}
$$

$\forall t \in I$. Hence, we conclude that $\dot{V}_{p}<0$ when $\left\|r_{p} \varepsilon_{p}\right\|>$ $\frac{M_{p} M_{v}}{k_{p} m_{p}}$. Since $r_{p_{i}}$ is positive definite, $\forall i \in\{1, \ldots, n\}$, the latter is equivalent to $\left\|\varepsilon_{p}\right\|>\frac{M_{p} M_{v}}{k_{p} m_{p} \underline{r}_{p}} \Rightarrow \dot{V}_{p}<0$. Hence, we conclude that all $\widetilde{x}(t) \in \mathfrak{X}$ satisfy

$$
\left\|\varepsilon_{p}(t)\right\| \leq \bar{\varepsilon}_{p}:=\max \left\{\left\|\varepsilon_{p}\left(t_{0}\right)\right\|, \frac{M_{p} M_{v}}{k_{p} m_{p} \underline{r}_{p}}\right\} .
$$

Since $\bar{\varepsilon}_{p}$ is finite, it holds that $T\left(\bar{\varepsilon}_{p}\right)<1$. Hence $\left|T\left(\varepsilon_{p_{i}}(t)\right)\right| \leq T\left(\bar{\varepsilon}_{p}\right)<1, \forall i \in\{1, \ldots, n\}, t \in I$. Moreover, since $T(\cdot)$ and $T^{-1}(\cdot)$ are smooth, the derivative $\frac{\partial T^{-1}(z)}{\partial z}$ approaches infinity only when $z \rightarrow \pm 1$. Therefore, in view of the definition of $r_{p_{i}}$ in (7), we conclude the existence of a finite $\bar{r}_{p}>0$ such that $\left\|r_{p}(t)\right\| \leq \bar{r}_{p}, \forall t \in I$. Next, (4) implies that $\left\|e_{p}(t)\right\| \leq \bar{e}_{p}:=M_{p} T\left(\bar{\varepsilon}_{p}\right) \sqrt{n}, \forall t \in I$. Hence, we conclude that $\left\|v_{\mathrm{d}}(t)\right\| \leq \bar{v}_{\mathrm{d}}:=\bar{x}_{\mathrm{d}}+\frac{M_{\dot{p}}}{m_{p}} \bar{e}_{p}+k_{p} \bar{r}_{p} \bar{\varepsilon}_{p}$, $\forall t \in I$, where $\bar{x}_{\mathrm{d}}$ is the uniform bound of the desired trajectory. We also conclude that $\|x(t)\| \leq \bar{x}:=\bar{e}_{p}+\bar{x}_{\mathrm{d}}$, $\forall t \in I$. In addition, by employing $\dot{x}=e_{v}+v_{\mathrm{d}}$ and (13), we conclude that $\|\dot{x}(t)\|<\widetilde{v}:=M_{v} \sqrt{n}+\bar{v}_{\mathrm{d}}, \forall t \in I$. Finally, by differentiating $v_{\mathrm{d}}$, employing the smoothness and boundedness of $\rho_{p}$ and its derivatives, the smoothness of $T(\cdot)$, the boundedness of $\ddot{x}_{\mathrm{d}}(t)$ as well as the aforementioned bounds, we can conclude the existence of a bound $\overline{d v_{\mathrm{d}}}$ such that $\left\|\dot{v}_{\mathrm{d}}(t)\right\|_{1} \leq \bar{d} v_{\mathrm{d}}, \forall t \in I$.

Note that, since $f(x, \dot{x})$ is Lebesgue measurable and locally bounded in $\mathbb{R}^{2 n}$ and $\|x(t)\| \leq \bar{x}<\infty,\|\dot{x}(t)\|<$ $\widetilde{v}<\infty, \forall t \in I$, there exists some positive $\bar{f}$ such that $\|f(x(t), \dot{x}(t))\|_{1} \leq \bar{f}, \forall t \in I$, and hence, for each $(x, \dot{x})$, since $\mathrm{K}[f]$ is formed by the convex closure of $f$, it holds that $\max _{z \in \mathrm{K}[f](x(t), \dot{x}(t))}\{z\} \leq \bar{f}, \forall t \in I$ and $\widetilde{x}(t) \in \mathfrak{X}$. Similarly, the continuity of $g(\cdot)$ implies that $\|g(x(t))\|_{1} \leq \bar{g}$ for a finite $\bar{g}, \forall t \in I$. Note that, in view of the aforementioned discussion, $\bar{f}$ and $\bar{g}$ depend solely on the initial conditions and the parameters of the funnel functions. Define now the finite constant term $d \in \mathbb{R}_{>0}$ as

$$
d:=\frac{\beta}{m_{v} \underline{m}}\left(\bar{f}+\bar{g}+\overline{d v_{\mathrm{d}}}+M_{\dot{p}} n\right),
$$

where $\underline{m}$ was introduced in Property 1 . Note that, in view of (13), the term in the parenthesis of (14) is an upper bound for the term $\| f(x(t), \dot{x}(t))+g(x(t))+\dot{v}_{\mathrm{d}}(t)+$ $\dot{\rho}_{v}(t) \rho_{v}(t)^{-1} e_{v}(t) \|_{1}, \forall t \in I$ and $\widetilde{x}(t) \in \mathfrak{X}$.

Define also the signal $\widetilde{d}:=\hat{d}-d$, where $\hat{d}$ is the adaptive gain introduced in (10), and consider the function

$$
V(\widetilde{\varepsilon}):=\alpha V_{p}+\frac{\beta}{2}\left\|\varepsilon_{v}\right\|^{2}+\frac{1}{2 \gamma_{\mathrm{d}}} \widetilde{d}^{2},
$$

where $\widetilde{\varepsilon}:=\left[\varepsilon_{p}^{\top}, \varepsilon_{v}^{\top}, \widetilde{d}\right]^{\top}$, and $\alpha>0$ is a positive constant to be defined; $V(\widetilde{\varepsilon})$ satisfies $W_{1}(\widetilde{\varepsilon}) \leq$ $V(\widetilde{\varepsilon}) \leq W_{2}(\widetilde{\varepsilon})$, for $W_{1}(\widetilde{\varepsilon}):=\min \left\{\frac{\alpha}{2}, \frac{\beta}{2}, \frac{1}{2 \gamma_{\mathrm{d}}}\right\}\|\widetilde{\varepsilon}\|^{2}$ and $W_{2}(\widetilde{\varepsilon}):=\max \left\{\frac{\alpha}{2}, \frac{\beta}{2}, \frac{1}{2 \gamma_{\mathrm{d}}}\right\}\|\widetilde{\varepsilon}\|^{2}$. Then, according to Lemma $1, \dot{V}(\widetilde{\varepsilon}(t)) \stackrel{\text { a.e. }}{\in} \dot{\tilde{V}}(\widetilde{\varepsilon}(t))$ with $\dot{\widetilde{V}}:=\bigcap_{\xi \in \partial V(\widetilde{\varepsilon})} \xi^{\top} \mathrm{K}[\dot{\vec{\varepsilon}}]$. Since $V(\widetilde{\varepsilon})$ is continuously differentiable, its generalized gradient reduces to the standard gradient and thus it holds 
that $\dot{\widetilde{V}}=\nabla V^{\top} \mathrm{K}[\dot{\tilde{\varepsilon}}]$, where $\nabla V=\left[\alpha \varepsilon_{p}^{\top}, \beta \varepsilon_{v}^{\top}, \frac{1}{\gamma_{d}} \widetilde{d}\right]^{\top}$. After using (2), (10), (11), and $x_{2}=v_{\mathrm{d}}+e_{v}$, one obtains

$$
\begin{aligned}
& \dot{\vec{V}} \subset \widetilde{W}_{s}:=-\alpha k_{p} \varepsilon_{p}^{\top} r_{p} \rho_{p}^{-1} r_{p} \varepsilon_{p}+\alpha \varepsilon_{p}^{\top} r_{p} \rho_{p}^{-1} e_{v}- \\
& \beta k_{v_{1}} \varepsilon_{v}^{\top} r_{v} \rho_{v}^{-1} M(x)^{-1} \rho_{v}^{-1} r_{v} \varepsilon_{v}+\widetilde{d}\left\|r_{v} \varepsilon_{v}\right\|_{1}- \\
& \beta \varepsilon_{v}^{\top} r_{v} \rho_{v}^{-1} M(x)^{-1}\left(\mathrm{~K}[f](x, \dot{x})+g(x)+\dot{v}_{\mathrm{d}}+\dot{\rho}_{v} \rho_{v}^{-1} e_{v}\right)- \\
& \beta k_{v_{2}} \varepsilon_{v}^{\top} r_{v} \rho_{v}^{-1} M(x)^{-1} \rho_{v}^{-1} \operatorname{SGN}\left(r_{v} \varepsilon_{v}\right)\left(k_{v_{3}}\left\|r_{p} \varepsilon_{p}\right\|_{1}+k_{v_{4}} \hat{d}\right) .
\end{aligned}
$$

Note that, since $\hat{d}\left(t_{0}\right) \geq 0$, (11) implies that $\hat{d}(t) \geq 0$, $\forall t \in I$. Moreover, since the Filippov regularization is defined as a closed set and $\dot{\tilde{V}} \subset \widetilde{W}_{s}$, it holds that $\max _{z \in \dot{\tilde{V}}}\{z\} \leq$ $\max _{z \in \widetilde{W}_{s}}\{z\}$. Therefore, in view of Assumption 1 and the definition of $d$ in (14), we obtain:

$$
\begin{aligned}
& \max _{z \in \tilde{\tilde{V}}}\{z\} \leq \max _{z \in \widetilde{W}_{s}}\{z\} \leq-\alpha \frac{k_{p}}{M_{p}}\left\|r_{p} \varepsilon_{p}\right\|^{2}-k_{v_{1}} \beta \underline{\lambda}\left\|r_{v} \varepsilon_{v}\right\|^{2}- \\
& k_{v_{2}} k_{v_{4}} \beta \underline{\lambda}\left\|r_{v} \varepsilon_{v}\right\|_{1} \hat{d}-k_{v_{2}} k_{v_{3}} \beta \underline{\lambda}\left\|r_{v} \varepsilon_{v}\right\|_{1}\left\|r_{p} \varepsilon_{p}\right\|_{1}+\widetilde{d}\left\|r_{v} \varepsilon_{v}\right\|_{1} \\
& +\left\|r_{v} \varepsilon_{v}\right\|_{1} d+\alpha\left\|\varepsilon_{p}^{\top} r_{p} \rho_{p}^{-1} e_{v}\right\|_{1},
\end{aligned}
$$

for all solutions $\widetilde{x}(t) \in \mathfrak{X}$. By setting $z=T\left(\varepsilon_{v_{i}}\right)$ in (6), we obtain $\left|T\left(\varepsilon_{v_{i}}\right)\right| \leq\left|r_{v_{i}} \varepsilon_{v_{i}}\right|$ and hence by employing $e_{v_{i}}=$ $\rho_{v_{i}} T\left(\varepsilon_{v_{i}}\right), i \in\{1, \ldots, n\}$, we obtain that

$$
\alpha\left\|\varepsilon_{p}^{\top} r_{p} \rho_{p}^{-1} e_{v}\right\|_{1} \leq \alpha \frac{M_{v}}{m_{p}}\left\|r_{p} \varepsilon_{p}\right\|_{1}\left\|r_{v} \varepsilon_{v}\right\|_{1} .
$$

Therefore, by setting $\alpha=\frac{k_{v_{2}} k_{v_{3}} m_{p} \beta \underline{\underline{\lambda}}}{M_{v}}$, employing $d=\hat{d}-\tilde{d}$, and in view of the fact that $\beta=\left(k_{v_{2}} k_{v_{4}} \underline{\lambda}\right)^{-1}$, we obtain

$$
\max _{z \in \tilde{\tilde{V}}}\{z\} \leq-\alpha \frac{k_{p}}{M_{p}}\left\|r_{p} \varepsilon_{p}\right\|^{2}-k_{v_{1}} \beta \underline{\lambda}\left\|r_{v} \varepsilon_{v}\right\|^{2}=: \quad-W(\widetilde{\varepsilon}),
$$

$\forall t \in I, \widetilde{x}(t) \in \mathfrak{X}$, where $W$ is continuous and positive semi-definite on $\mathbb{R}^{2 n+1}$, since $r_{v}$ and $r_{p}$ are positive definite. Hence, we conclude that $z \leq-W(\widetilde{\varepsilon}), \forall z \in \dot{\widetilde{V}}(\widetilde{\varepsilon}(t)), \forall t \in I$ and all $\widetilde{x}(t) \in \mathfrak{X}$. Choose now any finite $r>0$ and let $c<$ $\min _{\|\widetilde{\varepsilon}\|=r} W_{1}(\widetilde{\varepsilon})$. Note that all the conditions of Theorem 1 are satisfied and hence, all Filippov solutions starting from $\widetilde{\varepsilon}\left(t_{0}\right) \in \Omega_{f}:=\left\{\widetilde{\varepsilon} \in \mathcal{B}(0, r): W_{2}(\widetilde{\varepsilon}) \leq c\right\}$ are bounded and remain in $\Omega_{f}, \forall t \in I$. Moreover, $t_{\max }=\infty$, implying that $I=\left[t_{0}, \infty\right)$ and it also holds that $\lim _{t \rightarrow \infty}\left\|\varepsilon_{p}(t)\right\|=0$ and $\lim _{t \rightarrow \infty}\left\|\varepsilon_{v}(t)\right\|=0$, which, in view of the increasing property of $T(\cdot)$ and the fact that $T(0)=0$, implies that $\lim _{t \rightarrow \infty}\left\|e_{p}(t)\right\|=0$ and $\lim _{t \rightarrow \infty}\left\|e_{v}(t)\right\|=0$.

Note that $r$, and hence $c$, can be arbitrarily large allowing any finite initial condition $\widetilde{\varepsilon}$, which implies any $\left(\widetilde{x}\left(t_{0}\right), t_{0}\right) \in \mathcal{D}_{c}$. In addition, it holds that $\|\widetilde{\varepsilon}\|^{2} \leq \widetilde{c}:=$ $\left(\max \left\{\frac{\alpha}{2}, \frac{\beta}{2}, \frac{1}{2 \gamma_{\mathrm{d}}}\right\}\right)^{-1} c$, which implies the boundedness of $\left\|\varepsilon_{p}\right\|,\left\|\varepsilon_{v}\right\|$ and $d$ by $\sqrt{\widetilde{c}}$. Therefore, we conclude that $\|\hat{d}(t)\| \leq \bar{d}:=d+\sqrt{\widetilde{c}}, \forall t \in I$. Moreover, by employing (4), we conclude that $\left|\rho_{v_{i}}(t)^{-1} e_{v_{i}}(t)\right| \leq T(\sqrt{\widetilde{c}})<1$, and hence $\left|e_{v_{i}}(t)\right| \leq M_{v} T(\sqrt{\widetilde{c}}) \Rightarrow\left\|x_{2}(t)\right\| \leq \bar{x}_{2}:=M_{v} T(\sqrt{\widetilde{c}}) \sqrt{n}+$ $\bar{v}_{\mathrm{d}}, \forall t \in I$. Therefore, we conclude that all solutions are bounded in compact sets $\forall t \in I$, which means that $u$, and $\dot{\hat{d}}$, as designed in (10) and (11), respectively, remain also bounded, $\forall t \in I$.
Remark 3: Note that no boundedness assumptions or growth conditions are needed for the vector fields $f(x, \dot{x})$ and $g(x)$, whose effect is compensated by the introduced adaptive signal $\hat{d}$. Moreover, the response of the system is solely determined by the funnel functions $\rho_{p_{i}}$ and $\rho_{v_{i}}$, isolated from the system dynamics and the control gains selection. Nevertheless, appropriate gain tuning might be needed in order to suppress chattering due to the discontinuous nature of the proposed control scheme. In addition, note that the region of attraction (initial conditions) of $\left(\varepsilon_{p}, \varepsilon_{v}\right)=(0,0)$ is also isolated from the system dynamics and the control gain selection and depends only on the choice of the funnel functions $\rho_{p_{i}}, \forall i \in\{1, \ldots, n\}$. In particular, if $\rho_{p_{i}}\left(t_{0}\right)$ are design parameters, we can always choose them such that $-\rho_{p_{i}}\left(t_{0}\right)<e_{p_{i}}\left(t_{0}\right)<\rho_{p_{i}}\left(t_{0}\right), \forall i \in\{1, \ldots, n\}$, which renders the results global. In fact, the choice $\lim _{t \rightarrow t_{0}^{+}} \frac{1}{\rho_{p_{i}}\left(t_{0}\right)}=$ $0, \forall i \in\{1, \ldots, n\}$ [8] is not excluded from the control scheme and does not restrict the initial condition $x\left(t_{0}\right)$. Finally, unlike most related works, $\rho_{p_{i}}$ are not required to decrease to values arbitrarily close to zero, and asymptotic stability is still achieved. In fact, the proposed control schemes can be used to achieve simply asymptotic stability results without any funnel constraints, if the latter is not required. More specifically, given the initial errors $e_{p_{i}}\left(t_{0}\right)$, we can use the proposed control protocols by employing any constant values $\rho_{p_{i}}>\left|e_{p_{i}}\left(t_{0}\right)\right|, \forall i \in\{1, \ldots, n\}$.

\section{SIMULATION RESULTS}

We demonstrate the proposed control scheme using the dynamical model of a planar two-link robotic manipulator with $x=\left[x_{1}, x_{2}\right]^{\top} \in \mathbb{R}^{2}$, representing the angular joint positions; $f=\left[f_{1}, f_{2}\right]^{\top}$ is chosen as The respective Lagrangian dynamics are given by (2) with $M=\left[M_{i j}\right]$, $f=\left[f_{1}, f_{2}\right]^{\top}, g=\left[g_{1}, g_{2}\right]^{\top}, f_{1}=c_{1} \sin \left(x_{2}\right) \dot{x}_{1} \dot{x}_{2}+$ $c_{2} \dot{x}_{1} \operatorname{sgn}\left(\dot{x}_{1}\right)+c_{3} \dot{x}_{2}^{2}, f_{2}=c_{4} \sin \left(x_{2}\right) \dot{x}_{1}^{2}+c_{5} \dot{x}_{2} \operatorname{sgn}\left(\dot{x}_{2}\right)$, $g_{1}=g_{11} \cos \left(x_{1}\right)+g_{12} \cos \left(x_{1}+x_{2}\right), g_{2}=g_{21} \cos \left(x_{1}+x_{2}\right)$, and $m_{j}, c_{j}, g_{j k}$ are constant coefficients formed by inertial and geometrical characteristics of the robot (link lengths, masses, moments of inertia, friction coefficients). We set $x_{\mathrm{d}}=\left[2 \cos (t), \frac{\pi}{2}-2 \sin (t)\right]^{\top}, t_{0}=0, x_{1}(0)=[-2,2]^{\top}$, $x_{2}(0)=[0,0]^{\top}$, and the prescribed funnel functions as $\rho_{p_{i}}(t)=4.5 \exp (-0.25 t)+0.5, \forall i \in\{1,2\}$, which satisfy $\rho_{p_{i}}(0)>\left|x_{i}(0)-x_{\mathrm{d}_{i}}(0)\right|, \forall i \in\{1,2\}$ and converge to the value 0.5 . We also choose $\rho_{v_{i}}(t)=\left\|e_{v}(0)\right\|_{1} \exp (-0.1 t)+$ 0.5 , which satisfy $\rho_{v_{i}}(0)>\left|e_{v_{i}}(0)\right|, \forall i \in\{1,2\}$, as well as the gains $k_{p}=1, k_{v_{1}}=150, k_{v_{2}}=0.01, k_{v_{3}}=k_{v_{4}}=$ 0.5 , and $\gamma_{\mathrm{d}}=0.01$. The simulation results are depicted in Figs. 1,2 for $t \in[0,40]$ sec. More specifically, Fig. 1 shows the evolution of the transformed errors $\varepsilon_{p}(t), \varepsilon_{v}(t)$, $\forall t \in[0,40] \mathrm{sec}$, which, not also remain bounded, but also converge asymptotically to zero. Hence, one can conclude that $e_{p}(t)$ and $e_{v}(t)$ not only respect their imposed funnels but also converge asymptotically to zero, without the need of arbitrarily small values for $\lim _{t \rightarrow \infty} \rho_{p}(t)$ and $\lim _{t \rightarrow \infty} \rho_{v}(t)$. Finally, Fig. 2 illustrates the inputs $u(t)$ as well as the adaptation signal $\hat{d}(t), \forall t \in[0,40] \mathrm{sec}$. 

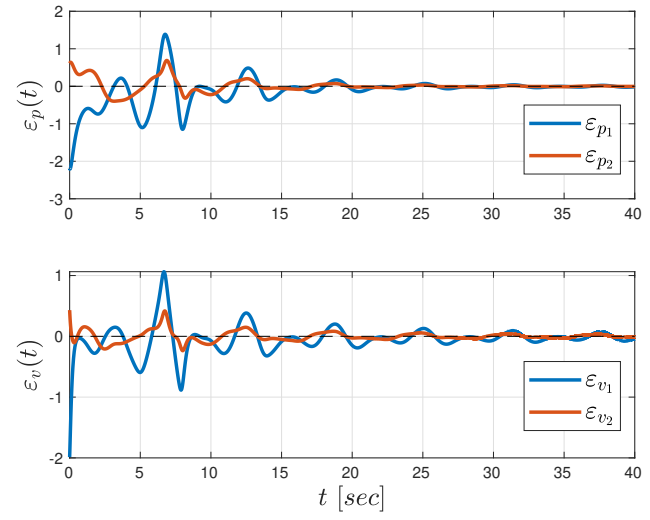

Fig. 1. The evolution of $\varepsilon_{p}(t), \varepsilon_{v}(t), \forall t \in[0,40]$ sec.
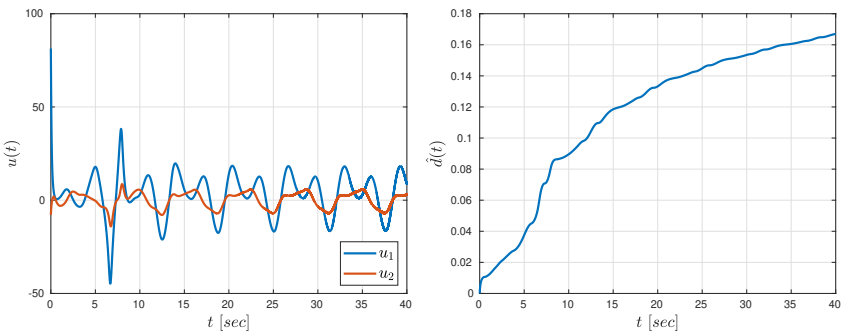

Fig. 2. The evolution of $u(t)=\left[u_{1}(t), u_{2}(t)\right]^{\top}$ (left) and $\hat{d}(t)$ (right), $\forall t \in[0,40]$ sec.

\section{CONCLUSION AND FUTURE WORK}

This paper tackles asymptotic stability subject to funnel constraints for 2nd-order nonlinear and uncertain Lagrangian systems. We design a control protocol based on adaptive and discontinuous control methodologies and the correctness of the proposed schemes is independent from the control gain selection. Future efforts will address more general systems of higher order with potential controllability relaxations.

\section{REFERENCES}

[1] S. S. Sastry and A. Isidori, "Adaptive control of linearizable systems," IEEE Transactions on Automatic Control, vol. 34, no. 11, 1989.

[2] J. Chen, A. Behal, and D. M. Dawson, "Robust feedback control for a class of uncertain mimo nonlinear systems," IEEE Transactions on Automatic Control, vol. 53, no. 2, 2008.

[3] Z. Wu, J. Chen, C. Wu, and K. Zhang, "Adaptive tracking for mimo nonlinear systems with unknown fast time-varying parameters," International Journal of Robust and Nonlinear Control, vol. 28, no. 13, pp. 4058-4074, 2018.

[4] L. Wang, R. Ortega, H. Su, and Z. Liu, "Stabilization of nonlinear systems nonlinearly depending on fast time-varying parameters: an immersion and invariance approach," IEEE Transactions on Automatic Control, vol. 60, no. 2, pp. 559-564, 2015.

[5] H. F. Grip, T. A. Johansen, L. Imsland, and G.-O. Kaasa, "Parameter estimation and compensation in systems with nonlinearly parameterized perturbations," Automatica, vol. 46, no. 1, pp. 19-28, 2010.

[6] C. P. Bechlioulis and G. A. Rovithakis, "Prescribed performance adaptive control for multi-input multi-output affine in the control nonlinear systems," IEEE Transactions on Automatic Control, vol. 55, no. 5, pp. 1220-1226, 2010.

[7] A. Theodorakopoulos and G. A. Rovithakis, "Low-complexity prescribed performance control of uncertain mimo feedback linearizable systems," IEEE Transactions on Automatic Control, vol. 61, no. 7, pp. 1946-1952, 2016.

[8] T. Berger, H. H. Lê, and T. Reis, "Funnel control for nonlinear systems with known strict relative degree," Automatica, vol. 87, 2018.
[9] A. Ilchmann and S. Trenn, "Input constrained funnel control with applications to chemical reactor models," Systems \& control letters, vol. 53, no. 5, pp. 361-375, 2004.

[10] Y. Karayiannidis and Z. Doulgeri, "Model-free robot joint position regulation and tracking with prescribed performance guarantees," Robotics and Autonomous Systems, vol. 60, no. 2, pp. 214-226, 2012.

[11] C. K. Verginis, M. Mastellaro, and D. V. Dimarogonas, "Robust cooperative manipulation without force/torque measurements: Control design and experiments," IEEE Transactions on Control Systems Technology, 2019.

[12] C. K. Verginis, C. P. Bechlioulis, D. V. Dimarogonas, and K. J. Kyriakopoulos, "Robust distributed control protocols for large vehicular platoons with prescribed transient and steady-state performance," IEEE Transactions on Control Systems Technology, vol. 26, no. 1, 2018.

[13] C. K. Verginis and D. V. Dimarogonas, "Timed abstractions for distributed cooperative manipulation," Autonomous Robots, vol. 42, no. 4, pp. 781-799, 2018.

[14] H. A. Hashim, S. El-Ferik, and F. L. Lewis, "Adaptive synchronisation of unknown nonlinear networked systems with prescribed performance," International Journal of Systems Science, vol. 48, no. 4, pp. 885-898, 2017.

[15] L. Macellari, Y. Karayiannidis, and D. V. Dimarogonas, "Multi-agent second order average consensus with prescribed transient behavior," IEEE Transactions on Automatic Control, vol. 62, no. 10, 2017.

[16] C. K. Verginis, A. Nikou, and D. V. Dimarogonas, "Robust formation control in se for tree-graph structures with prescribed transient and steady state performance," Automatica, 2019.

[17] A. Ilchmann and E. P. Ryan, "Asymptotic tracking with prescribed transient behaviour for linear systems," International Journal of Control, vol. 79, no. 8, pp. 910-917, 2006.

[18] A. Ilchmann, E. P. Ryan, and P. Townsend, "Tracking with prescribed transient behavior for nonlinear systems of known relative degree," SIAM Journal on Control and Optimization, vol. 46, no. 1, 2007.

[19] D. Liberzon and S. Trenn, "The bang-bang funnel controller for uncertain nonlinear systems with arbitrary relative degree," IEEE Transactions on Automatic Control, vol. 58, no. 12, 2013.

[20] Y. Wang, J. Hu, J. Li, and B. Liu, "Improved prescribed performance control for nonaffine pure-feedback systems with input saturation," International Journal of Robust and Nonlinear Control, 2019.

[21] M. Dresscher and B. Jayawardhana, "Prescribing transient and asymptotic behaviour of lti systems with stochastic initial conditions," IFACPapersOnLine, vol. 50, no. 1, pp. 1822-1827, 2017.

[22] Z.-P. Jiang, "Robust exponential regulation of nonholonomic systems with uncertainties," Automatica, vol. 36, no. 2, pp. 189-209, 2000.

[23] A. Astolfi, "Discontinuous control of nonholonomic systems," Systems \& control letters, vol. 27, no. 1, pp. 37-45, 1996.

[24] A. M. Bloch, M. Reyhanoglu, and N. H. McClamroch, "Control and stabilization of nonholonomic dynamic systems," IEEE Transactions on Automatic control, vol. 37, no. 11, pp. 1746-1757, 1992.

[25] F. Ceragioli and C. De Persis, "Discontinuous stabilization of nonlinear systems: Quantized and switching controls," Systems \& control letters, vol. 56, no. 7-8, pp. 461-473, 2007.

[26] K. D. Do and J. Pan, "Adaptive global stabilization of nonholonomic systems with strong nonlinear drifts," Systems \& Control Letters, vol. 46, no. 3, pp. 195-205, 2002.

[27] C. K. Verginis and D. V. Dimarogonas, "Adaptive leader-follower coordination of lagrangian multi-agent systems under transient constraints," Conference on Decision and Control, 2019, Accepted.

[28] C. K. Verginis and D. V. Dimarogonas, "Closed-form barrier functions for multi-agent ellipsoidal systems with uncertain lagrangian dynamics," IEEE Control Systems Letters, vol. 3, no. 3, pp. 727-732, 2019.

[29] B. Paden and S. Sastry, "A calculus for computing filippov's differential inclusion with application to the variable structure control of robot manipulators," IEEE transactions on circuits and systems, vol. 34, no. 1 , pp. 73-82, 1987.

[30] N. Fischer, R. Kamalapurkar, and W. E. Dixon, "Lasalle-yoshizawa corollaries for nonsmooth systems," IEEE Transactions on Automatic Control, vol. 58, no. 9, pp. 2333-2338, 2013.

[31] C. P. Bechlioulis and G. A. Rovithakis, "A low-complexity global approximation-free control scheme with prescribed performance for unknown pure feedback systems," Automatica, vol. 50, no. 4, pp. 1217-1226, 2014.

[32] J. Cortes, "Discontinuous dynamical systems," IEEE control Systems, vol. 28 , no. 3,2008 . 